\title{
Consideration about long-term process of dental pulp conservative treatment for the coronal pulp widely replaced with hard tissue
}

\author{
Kayoko Kitajima* \\ Department of Endodontics, School of Life Dentistry at Niigata, The Nippon Dental University, 1-8 Hamaura-cho, Chuo-ku, Niigata, Niigata 951-8580, Japan
}

\begin{abstract}
Two cases that involved deep caries or abrasion, but making abundant reparative dentin were described. In one case, these deep caries were removed softened dentin completely by round bur and spoon excavator. In another case, calcified pulp cavity was exposed when the tooth was prepared for crown formation. These dentin surfaces were observed. Both teeth had no pain, bleeding and those impedance measurements indicated no pulp exposure values. Surface of dentin was irrigated enough of $7 \% \mathrm{NaClO}$, indirect pulp capping with $\mathrm{Ca}(\mathrm{OH})_{2}$, was performed on it and lined with base cement under rubber dam isolation. Finally, those teeth were repaired with composite resin or set with a crown after following up for three weeks. In both cases, clinical course has remained excellent after treatment for 12 and 17 years.
\end{abstract}

\section{Introduction}

The disappearance of the root canal on a dental X-ray photograph is associated with the calcification of the pulp tissue and calcified deposits on the root canal wall, both of which are often attributed to aging [1-10].

In recent years, the lifetime of teeth has been extended with trends such as improvements in oral hygiene, advances in dental prophylaxis, and the promotion of tooth preservation. There have also been an increasing number of cases wherein root canal detection is difficult to perform because a growing number of teeth have recently demonstrated a tendency of having marked levels of calcification at the root canal orifice, stenosis, and closure as a result of encouraging all individuals, regardless of their age, to brush. Furthermore, with the advent of an aging society, the stenotic tendency is expected to accelerate further due to aging-related changes in the root canal as well as factors such as long-term mechanical, cold/heat, and chemical stimulation; dental caries; and periodontal disease. Endodontic treatment for such teeth is extremely difficult. However, the success of this treatment will most likely reflect the manner in which we would address subsequent prosthetic treatments, as well as the need for biting with one's own teeth for as long a period as possible in one's life. We should avoid completing unnecessary root canal treatments and instead, increase the success rate of necessary treatments, including prognosis. To this end, it is crucial to correctly determine whether endodontic treatment is a necessity.

We herein report our recent experiences involving two patients with deep cavities for whom pulpectomy should essentially have been selected. However, due to marked calcified deposits in pulp cavities, the patients instead underwent preservation treatment of pulp and followed excellent clinical courses.

\section{Case 1}

Patient: A 51-year-old Japanese male

Chief complaint: The patient visited our hospital hoping to have composite resin on the maxillary right and left central incisors restored, which had previously detached and been restored.

Systemic medical history and family history: unremarkable

Present illness: The patient had previously undergone tooth repair 4 or 5 years before and had experienced no symptoms since; however, he was concerned regarding the recently observed discoloration of the restorative dental materials. The dental restorative material had detached 1 week before.

Findings of physical examination: A detachment of the class IVcomposite resin restoration was observed in areas including the mesial angles of the maxillary right and left central incisors.

The patient experienced no spontaneous pain nor induced pains such as cold- and heat-induced pain, pain on vertical and horizontal percussion, and occlusal pain. No abnormal findings were observed in the gingiva. X-ray photographs showed transmission images of deep caries as well as pulp horn retraction (Figure 1-A). There were no transmission images at the apical area. Pulp electro diagnosis showed vital reactions.

${ }^{*}$ Correspondence to: Kayoko Kitajima, Department of Endodontics, School of Life Dentistry at Niigata, The Nippon Dental University, 1-8 Hamaura-cho, Chuo-ku, Niigata, Niigata 951-8580, Japan, E-mail: kitajima@ngt.ndu.ac.jp

Key words: dental pulp, calcification, aging, abrasion

Received: December 19, 2019; Accepted: December 26, 2019; Published: December 30, 2019 


\section{Diagnosis: Class 2 caries}

Treatment: A rubber dam isolation was applied to this tooth, and all composite resin restorative materials were removed. The carious portion dyed with a caries detector dye was cut away carefully using a round bur and excavator. Careful probing of the cavity floor using a rounded probe detected no bleeding; hard dentin was palpated so that the patient felt no severe pain, even in an anaesthetized state. Impedance measurements showed no pulp exposure values (Figure 1-B,C).The dentin was washed with a solution of $7 \% \mathrm{NaClO}$ and $3 \% \mathrm{H}_{2} \mathrm{O}_{2}$; after drying, indirect pulp capping was performed using a calcium hydroxide preparation, followed by completing the composite resin restoration.

Clinical course: The patient visited again after 7 years from first visit, because the color of the composite resin restoration materials changed. The patient hoped to have more repair done.

Hard tissue formation was confirmed under indirect pulp capping materials (Figure 1-D, E). Approximately 12 years have passed after the first treatment, but the patient has experienced no spontaneous pain nor any of the induced pains mentioned above. With no abnormal findings observed in the peripheral tissues and X-ray findings (Figure1-F), his clinical course has remained excellent.

\section{Case 2}

Patient: A 67-year-old Japanese woman.

Chief complaint: The patient visited our hospital for a facing crown placed on her maxillary left central incisor to improve its poor aesthetics.

\section{Systemic medical history and family history: Unremarkable}

Present illness: The patient had received a facing crown on the maxillary right central incisor over 10 years prior at the other dental clinic, but it had detached and been lost 1 week prior to this first visit. (Figure
A

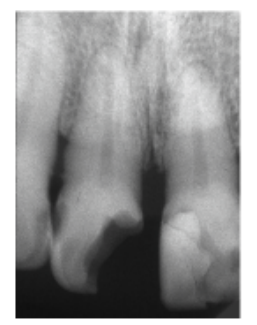

D

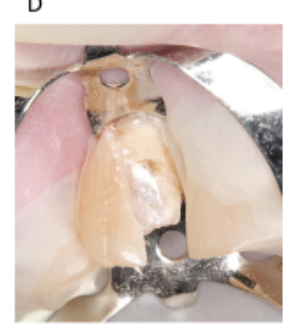

B

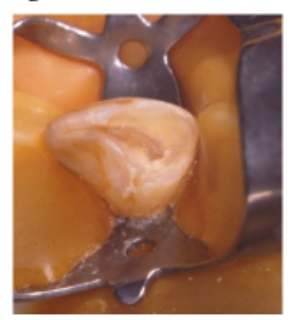

E

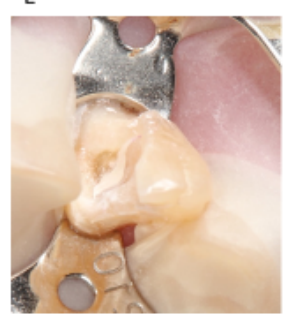

C

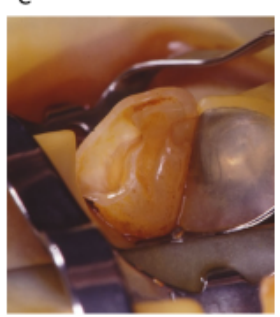

F

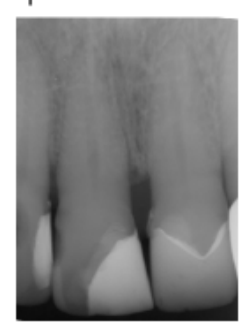

Figure 1.

A : 1 1 11 X-ray finding at the first visit of case 1

B : 1] Oral view when softened dentin was removed

C : 1 1 Oral view when softened dentin was removed

D : 1] Oral view when the secondary treatment

$\mathrm{E}: 11 \quad$ Oral view when the secondary treatment

$\mathrm{F}: \underline{1} 11$ X-ray finding at the 12 years later from first visit
A

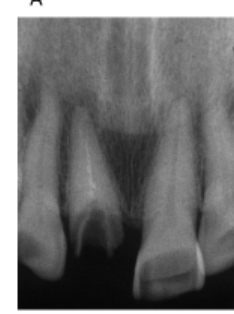

D

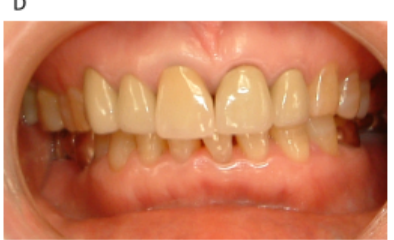

Figure 2.

A : $1|1| 1$ X-ray finding at the first visit of case 2

B : 11 Oral view when softened dentin was removed and prepared crown formation

C : 1 Oral view after indirect pulp capping was performed and removed rubber dam isolation

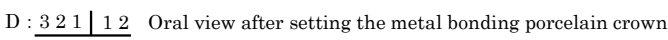

E : 1 Oral view after extracted du to root fracture

1 No problem

$\mathrm{F}: \underline{21} 11$ X-ray finding at 5 years later after setting bridge and at 17 years later from first visit

1 No problem

2-A); therefore, she had a new metal bonding porcelain crown prepared and placed following a root canal treatment. She subsequently began being concerned regarding the poor aesthetics of the left central incisor and started wanting a new metal bonding porcelain crown prepared for it as well, so as to look the same as the right central incisor. Although she was concerned about its poor color, she had left the left central incisor unattended to because of the absence of any pain.

Findings of physical examination: The X-ray photographs revealed that the enamel of the maxillary left central incisor had worn and the tooth substance had become thinner. As a result, the radiolucency of half of the tooth area toward the cutting edge away from the cervix was enhanced (Figure 2-A). Discoloration due to exposure of the dentin was observed. The patient experienced no spontaneous pain nor induced pains such as cold- or heat, pain on vertical and horizontal percussion, and occlusal pain. No abnormal findings were observed in the gingiva. Pulp electro diagnosis showed vital reactions.

\section{Diagnosis: Abrasion and class 2 caries}

Treatment: After initiating abutment tooth preparation for the metal bonding porcelain crown, it was found that the dentin had been replaced with yellowish-brown hard tissue, in which the anatomical form of the original pulp cavity appeared different from that of the dentin. After carefully removing the lesion dyed with a caries detector dye using a round bar, an adjustment of the abutment tooth form was performed. The yellowish-brown hard tissue replaced within the original pulp cavity was carefully probed but detected no bleeding; the hard tissue was palpated so that the patient felt no severe pain, even in an unanaesthetized state (Figure 2-B). Impedance measurement showed no pulp exposure values. A rubber dam isolation was applied to the tooth. The tooth was washed with a solution of $7 \% \mathrm{NaClO}$ and $3 \% \mathrm{H}_{2} \mathrm{O}_{2}$, and after drying, indirect pulp capping using a calcium hydroxide preparation was performed (Figure 2-C), followed by placing a temporary crown. During a 3-week follow-up period, no 
symptoms were observed. After 3 weeks impression-taking and bitetaking for a metal bonding porcelain crown were completed and subsequently one as requested for the patient was placed. After treating the left central incisor, the metal bonding porcelain crowns were set up at the left lateral incisor, right lateral incisor and right canine by patient's requests (Figure 2-D). After 12years from first visit, a root fracture of right central incisor happened and it was extracted. The right lateral incisor and canine, and the left central incisor were prepared as an abutment of bridge. The left central incisor was no problem and in good condition at that time (Figure 2-E).

Over a long-term follow-up period of approximately 17 years after the metal bonding porcelain crown placement on the left central incisor, the patient experienced no spontaneous pain or induced pain such as cold- or heat, pain on percussion, and occlusal pain. With no abnormal findings observed in the gingiva and peripheral periodontal tissues (Figure 2-F), her clinical course was excellent.

\section{Discussion}

The average lifespan of the Japanese in 2018 was 81.25 years for men and 87.32 years for women [11]. The men's record has been broken for 6 consecutive years, and the women's one has been broken for 7 consecutive years. The world's top five countries for longevity in men are Hong Kong, Switzerland, Japan, Norway, and Sweden, where men's average lifespan is over 80 years. On the other hand, those for longevity in women are Hong Kong, Japan, Spain, Korea, and Switzerland, where women's average lifespan is over 85 years. Such advances may be attributed to the results of improvements in medicine and people's health consciousness, and the average lifespan is likely to expand even further. With the extension of the human lifespan, the life of teeth has similarly extended, giving rise to the unprecedented changes that have begun to occur in them. These changes include the addition of secondary dentin due to aging and the addition of reparative dentin and ectopic calcification due to extended and increased opportunities for contact with exogenous stimuli [1-10,1221]. Such changes eventually result in calcareous deposition on the root canal wall and pulp cavity stenosis and narrowing, thereby inducing a strong tendency of root canal closure. The crown and the cervix in particular are susceptible to pulp horn retraction, pulp cavity stenosis, and root canal narrowing, associated with a wide range of factors such as wear due to attrition and excessive brushing, cold, and heat stimulation due to gingival retraction, tooth root exposure, and root caries development.
A

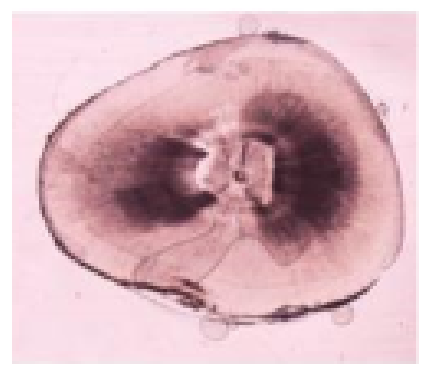

B

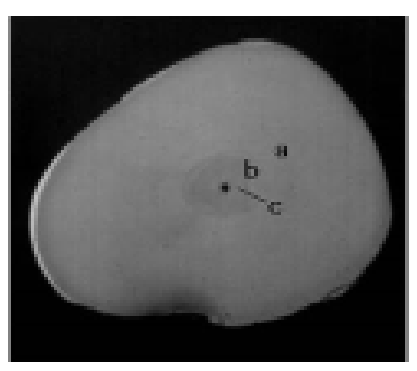

Figure 3.

A Transmitted light (Cross section)of human lower left central incisor

B Contact Micro Radiogram (Cross section) of same section

a primary dentin

b secondary dentin

c narrowed root canal
In Figure 3-A,B, the extracted tooth where the root canal could not be confirmed in X-ray photographs was horizontally cut. It was then observed by CMR (Contact micro radiogram) that the calcification strongly appears particularly in the vicinity of the tooth and neck by the addition of the second dentin and repair as compared with the vicinity of the apical portion. It shows that the root canal diameter is extremely small [22].

However, in teeth that have caused the narrowing and closure of the root canal in such a manner, root canal treatment becomes extremely difficult and the prognosis is poor when the treatment is not sufficiently successful. That is, once having initiated the root canal treatment, mechanical root canal enlargement formation, and chemical cleaning and bonding, it is a standard rule that a close three-dimensional root canal filling is completed. However, to mechanically expand and form the narrowed root canal to achieve physiological root palpitations, advanced techniques are required. In some cases, because of step formation and perforation in the middle, the root canal may cause the therapeutic instrument to break off while performing unreasonable expansion formation. In such a scenario, sterile treatment becomes extremely difficult because it becomes impossible to follow the original root canal, and there is the high risk for poor prognosis.

The two cases reported herein are significant examples of calcification. Despite the deep caries and veto tooth formation being performed, a good course over a long period of time without root canal treatment was followed and the pulp could be saved.

The main point wherein one should be careful in such cases is for appropriately determining the applicability of sterile treatment. First, the vicinity should be searched for any sites that show the value of pulp exposure in the caries and the surface of prepared teeth. Chemical washing using an organic substance dissolving agent under rubber dam isolation technique and drying were performed. Indirect pulp capping was performed with an indirect pulp capping agent to prevent coronal leakage just in case. Repair dentin is prone to be rougher than native dentin. The two cases reported herein were not pulp exposure, but Goldberg, et al. [23] reported that they performed pulpotomy, pulp capping with $\mathrm{Ca}(\mathrm{OH})_{2}$ directly and observed dentin bridge formation using scanning electron microscope (SEM). In consequence of that, they indicated there are many holes and defects in the dentin bridge.

Therefore, it is necessary to consider the risk for this evolving into a means of infection passage. It is preferable that long-term follow-up be completed for this reason. These two cases were able to observe good condition by X-Ray for 12 and 17 years after treatment.

In case 2, wear was observed in the enamel of the labial side over a large number of teeth in the oral cavity (Figure 2-B). From this finding, the substitution of the high calcification in the upper left central incisor pulp cavity may have been caused by a long-term mechanical stimulation. It seems to be repair mechanism with less holes and defects. Therefore, it is the reason why vital left central incisor has been maintained and performed for long period after right central incisor was extracted and it became an abutment tooth of bridge.

Due to a longer lifespan in humans and the related elongation of the life of the teeth, it is considered that the crown of the tooth and the tooth neck can become highly calcified, as shown in these examples. It may be possible to achieve preservation of teeth over a long period of time provided that coarse tissue representing an infection path is addressed by striving to prevent non-sterile treatment and coronal leakage, and difficult root canal treatment can be avoided by the application of pulp preservation therapy. 


\section{Conclusion}

In cases where an X-ray transmission image that casts suspicion for conditions such as deep caries or abrasion in the crown, it is important to determine the appropriate treatment, considering the formation of the repair dentin and based on the depth of the fossa and the surface of tooth preparation. It is important to accurately determine whether the preservation of the pulp is possible. When starting the root canal treatment, it is necessary to re-recognize that fulfilling the three main principles of endodontic treatment becomes an absolute requirement. In particular, in teeth with a stenosis tendency of the pulp cavity and root canal, it is important to choose the treatment only after sufficient testing and examinations.

\section{References}

1. Nalbandian J, Gonzales F, Sognnaes RF (1960) Sclerotic age changes in root dentin of human teeth as observed by optical, electron, and x-ray microscopy. J Dent Res 39: 598-607. [Crossref]

2. Pinzon RD, Toto PD, O'Malley JJ (1966) Kinetics of rat molar pulp cells at various ages. J Dent Res 45: 934-938. [Crossref]

3. Sayegh FS, Reed AJ (1968) Calcification in the dental pulp. Oral Surg Oral Med Oral Pathol 25: 873-882. [Crossref]

4. Philippas GG, Applebaum E (1968) Location of irregular secondary dentin formation. $J$ Dent Res 47: 769-778. [Crossref]

5. Avery JK (1972) Dentin. Pulp. In: Baskar SN (Ed.) Orbanâ $€^{\mathrm{TM}_{\mathrm{S}}}$ oral histology and embryology 9 ed, CV Mosby, St Louis: 107-179.

6. Seltzer S, Bender IB (1983) The Dental Pulp biologic consideration in dental procedure 3 ed, JB Lippincott, Philadelphia: 324-348.

7. Nitzan DW, Michaeli Y, Weinreb M, Azaz B (1986) The effect of aging on tooth morphology: a study on impacted teeth. Oral Surg Oral Med Oral Pathol 61: 54-60. [Crossref]

8. Morse DR (1991) Age-related changes of the dental pulp complex and their relationship to systemic aging. Oral Surg Oral Med Oral Pathol 72: 721-745. [Crossref]
9. Ten Cate AR (1994) Oral histrogy, development, structure and function 4 ed, CV Mosby, St Louis: 147-217.

10. Hargreaves KM, Cohen S (2011) Cohenâ $€^{\mathrm{TM}_{\mathrm{S}}}$ Pathology of the Pulp. 10 ed. Mosby Co, Elesevier, 489-503.

11. https://www.who.int/gho/mortality_burden_disease/life_tables/situation_trends/en/

12. Beust TB (1931) Physiologic changes in the dentin. J Dent Res 11: 267.

13. Takuma S, Eda S (1965) Further studies on the peritubular matrix in dentin. J Dent Res 44:1148-1149.

14. Bergman G, engfeldt B (1965) Studies on mineralized dental tissues. 11. Microradiography as a method for studying dental tissues and its application to the study of caries. Acta Odontol Scand 23: 197.

15. Berggren H (1965) The reaction of the translucent zone of dentine to dyes and radioisotopes. Acta Odontol Scand 23: 197-213. [Crossref]

16. Takuma S, Sunohara H, Watanabe H, Yama K (1969) Some structural aspects of carious lesions in human dentin. Bull Tokyo Dent Coll 10: 173-181. [Crossref]

17. Tronstad L, Langeland K (1971) Effect of attrition on subjacent dentin and pulp. J Dent Res 50: 1-30. [Crossref]

18. Aoyama I, Katagiri M, Suga S (1972) Ultrastructure of sound and sclerosed dentin tubules viewed by scanning electron microscopy. in: Proceedings of the 6th International Conference on X-ray optics and Microanalysis; University of Tokyo Press, Tokyo: 867-871.

19. Tronstad L (1978) Vital staining of coronal dentin in monkey teeth. Oral Surg Oral Med Oral Pathol 45: 612-616. [Crossref]

20. Brännström M, Garberoglio R (1980) Occlusion of dentinal tubules under superficial attrited dentine. Swed Dent J 4: 87-91. [Crossref]

21. Saruwatari R, Ban S, Ohno (2000) Characterization of apatite in the translucent dentin of single root. Aichi-Gakuin Dent Sci 13: 37-46.

22. Kitajima K, Tanaka M, Miyoshi T, Igarashi M, Kawasaki K (2006) Histological observations of the structure of human mandibular incisors with obstructed pulp chamber and canal in radiographs. Jap J Conserv Dent 49: 867-876.

23. Goldberg F, Massone EJ, Spielberg C (1984) Evaluation of the Dental Bridge after pulpotomy and calcium Hydroxide dressing. J of Endod 10: 318-320.

Copyright: (C2019 Kitajima K. This is an open-access article distributed under the terms of the Creative Commons Attribution License, which permits unrestricted use, distribution, and reproduction in any medium, provided the original author and source are credited. 